\title{
Continuous Inertial Focusing and Separation of Particles by Shape
}

\author{
Mahdokht Masaeli, ${ }^{1,2, *}$ Elodie Sollier, ${ }^{1,2, \dagger}$ Hamed Amini, ${ }^{1,2}$ Wenbin Mao, ${ }^{3}$ Kathryn Camacho, ${ }^{4}$ Nishit Doshi, ${ }^{4}$ \\ Samir Mitragotri, ${ }^{4}$ Alexander Alexeev, ${ }^{3}$ and Dino Di Carlo ${ }^{1,2, \$}$ \\ ${ }^{1}$ Department of Bioengineering, University of California, Los Angeles, 410 Westwood Plaza, Los Angeles 90095, California, USA \\ ${ }^{2}$ California NanoSystems Institute, University of California, Los Angeles 90095, California, USA \\ ${ }^{3}$ Woodruff School of Mechanical Engineering, Georgia Institute of Technology, 771 Ferst Drive Northwest, \\ Atlanta 30332, Georgia, USA \\ ${ }^{4}$ Department of Chemical Engineering, University of California, Santa Barbara,, Mail Code 5080, \\ Santa Barbara 93106, California, USA
}

(Received 20 March 2012; revised manuscript received 15 May 2012; published 12 September 2012)

\begin{abstract}
An effective approach to separating shaped particles is needed to isolate disease-causing cells for diagnostics or to aid in purifying nonspherical particles in applications ranging from food science to drug delivery. However, the separation of shaped particles is generally challenging, since nonspherical particles can freely rotate and present different faces while being sorted. We experimentally and numerically show that inertial fluid-dynamic effects allow for shape-dependent separation of flowing particles. (Spheres and rods with aspect ratios of 3:1 and 5:1 have all been separable.) Particle rotation around a conserved axis following Jeffery orbits is found to be a necessary component in producing different equilibrium positions across the channel that depend on particle rotational diameter. These differences are large enough to enable passive, continuous, high-purity, high-throughput, and shape-based separation downstream. Furthermore, we show that this shape-based separation can be applied to a large range of particle sizes and types, including small, artificially made $3-\mu \mathrm{m}$ particles as well as bioparticles such as yeast. This practical approach for sorting particles by a previously inaccessible geometric parameter opens up a new capability that should find use in a range of fields.
\end{abstract}

DOI: 10.1103/PhysRevX.2.031017 Subject Areas: Biological Physics, Fluid Dynamics, Interdisciplinary Physics

\section{INTRODUCTION}

Shape represents one of the most important factors for specifically identifying a bioparticle [1]. Among other factors, shape is a marker of cell cycle. For example, eukaryotic cells such as yeast show cell-cycle-dependent changes in their shape as a budding daughter cell forms attached to the mother cell [2]. Shape is also an indicator of cell state useful in clinical diagnostics. For example, changes of red blood cells from their normal biconcave shapes to other shapes accompany many diseases, such as sickle-cell disease, anamia, or malaria [3,4]. Thus, shape can be a specific marker in bioparticle separation and may serve as a useful new basis for passive particle fractionation. More generally, many particles of interest such as parasites, bacteria, viruses, but also marine organisms $[5,6]$ and manmade microparticles like barcoded substrates $[7,8]$ or cement microparticles, possess a variety of shapes, and

\footnotetext{
*Corresponding author. mahdokhtm@ucla.edu

${ }^{\dagger}$ Corresponding author. esollier@ucla.edu

${ }^{\ddagger}$ Corresponding author. dicarlo@seas.ucla.edu
}

Published by the American Physical Society under the terms of the Creative Commons Attribution 3.0 License. Further distribution of this work must maintain attribution to the author(s) and the published article's title, journal citation, and DOI. the ability to prepare particular shapes at high purities enables clinical, industrial, and research applications.

Given that traditional pore-based filtration is ineffective with bioparticles that may be deformable or may possess unique shapes, a diverse set of methods has recently emerged to achieve continuous separation of cells and microparticles $[9,10]$. Some approaches combine microfluidic flow with a force field, such as electric [11], magnetic [12,13], optical [14], or acoustic [15] fields, or with biochemical interactions (selective lysis or antigenantibody capture). Other approaches are based on passive hydrodynamics in microchannels, for example, microfiltration [16]; pinched-flow fractionation (PFF) and hydrodynamic filtration (HDF) [17,18]; hydrophoresis [19-24]; deterministic lateral displacement (DLD) around pillars [25]; and inertial separation in curved channels or spirals [26-28].

However, particle shape has not been considered in most of these separation techniques. Only recently have researchers investigated HDF, DLD, and dielectrophoresis (DEP) for shape-based separations. Beech et al. first recognized the need to separate shaped parasites from blood and implemented an approach using the DLD technique. DLD devices consist of networks of spatially distributed posts in which spatial arrangement defines a size cutoff (or critical radius) for separation. DLD has been demonstrated to enable a separation based on particle morphology, via control of particle orientation, or particle deformability, via control of 
shear stress [29]. Holm et al. applied this technique for the separation of trypanosomes from red blood cells [30] and for the classification of morphologically altered red cells [29]. More recently, Sugaya et al. applied HDF for shapebased sorting of budding and single cells from a yeast-cell mixture [31]. HDF, which employs highly branched channel structures, is based on differential particle behavior in a fluid branch point due to steric exclusion, with the size cutoff for separation being determined by the fluidic resistance ratio of the channel that defines the amount of the fluid that splits into each channel. Similar to the mechanism underlying DLD, the rotation of nonspherical particles in HDF modifies their effective steric dimension. Both HDF and DLD are efficient, passive, and continuous techniques, but both require (i) highly complex features-130 branch channels for HDF [31], and complex and high-resolution arrays of posts with 13 different arrangements for DLD [29], and (ii) low flow rates $-60 \mathrm{~nL} / \mathrm{min}$ for Holm et al. [30] and 2-3 $\mu \mathrm{L} / \min$ for Sugaya et al. [31], consequently offering a low throughput that may be suitable for research applications but not for industrial-scale applications. Similarly, Valero et al. performed shape-based sorting of yeast by balancing opposing DEP forces at multiple frequencies [32]. DEP requires the integration of active elements and a precise and reproducible control of the buffer conductivity between each experiment; both of these requirements complicate potential use beyond research applications.

Recently, Di Carlo and others have shown that inertial focusing, based on effects of fluid inertia on migration in cylindrical pipes first observed by Segre and Silberberg [33], can be used to separate microparticles and cells in microchannels at high rates [28,34-36]. Briefly, two inertial lift forces are involved: (i) a shear-gradient lift force and (ii) a wall-effect lift force induce particle migration across streamlines when the Reynolds number of the particle, $R e_{\mathrm{p}}$, is of order 1 or greater. $\operatorname{Re}_{\mathrm{p}}=\operatorname{Re}_{\mathrm{e}}(a / W)^{2}$ with $a / W$ being the ratio of particle to channel diameter, and $R e_{\mathrm{e}}$ indicates the Reynolds number for the channel flow, $R e_{\mathrm{e}}=$ $\rho U_{\mathrm{m}} W / \mu$. Here, $\rho, U_{\mathrm{m}}$, and $\mu$ correspond to the density, maximum velocity, and dynamic viscosity of the fluid. In rectangular or square channels, particles generally migrate to two to four distinct dynamic equilibrium positions depending on the fold symmetry of the channel cross section (Fig. 1). Among other advantages, the possibility of operating over a large range of high flow rates makes inertial focusing a promising technique for low-cost cytometry, massively parallel cell separation, and washing [37-39]. However, previous work has mainly investigated spherical particles and characterized the ability of inertial focusing to separate or focus particles or cells based on the overall particle diameter [28]. Recently, Hur et al. demonstrated deformability-based differences in equilibrium positions (stable focusing positions in the channel) for cells and viscous droplets [40]. Some of the current authors also showed that inertial effects can be exploited to focus
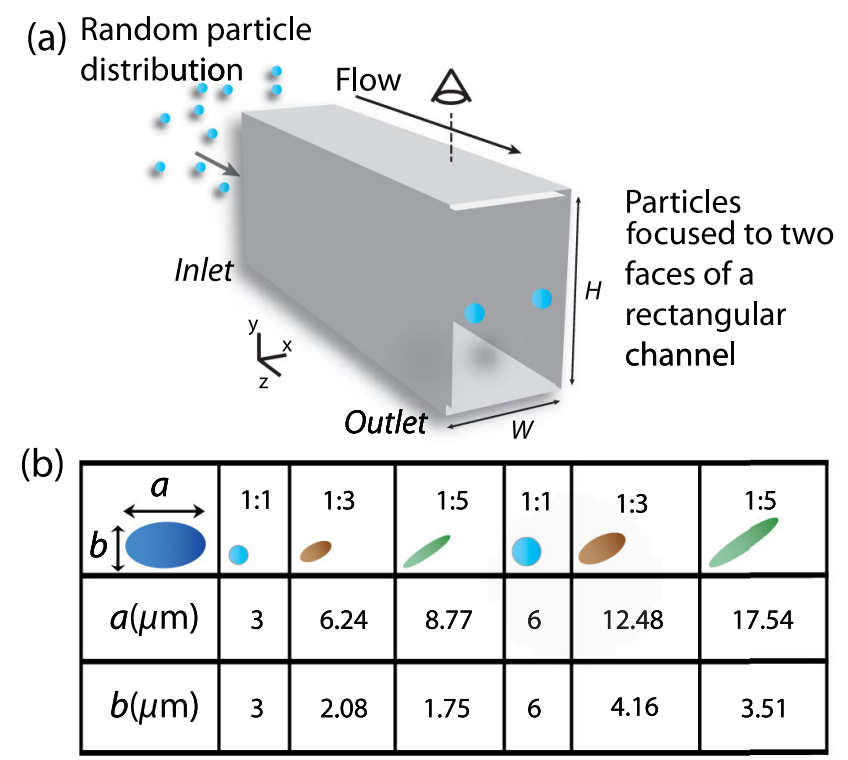

(c)
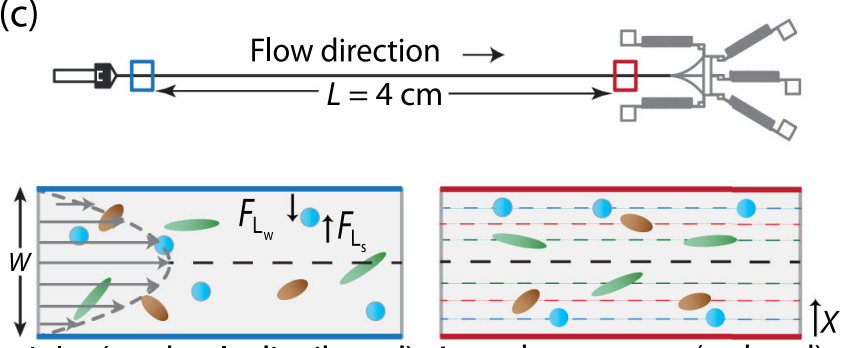

Inlet (randomly distributed)
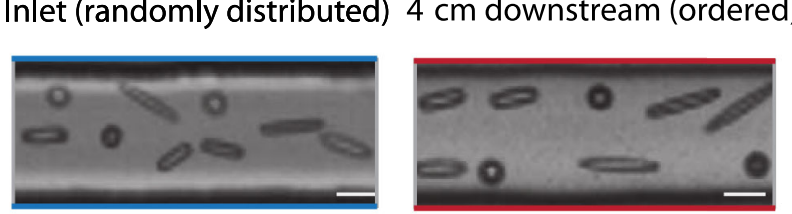

- - Channel center

Scale bar: $10 \mu \mathrm{m}$

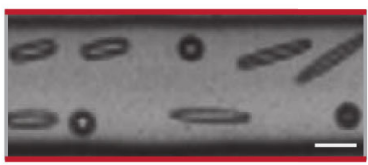

$X_{\text {eq }}=50\left(1-\left|\frac{2 X}{W}-1\right|\right)$

FIG. 1. Focusing of ellipsoids of different aspect ratios to two sets of dynamic equilibrium positions. (a) In rectangular channels with a high aspect ratio, at moderate $R e$, randomly distributed particles are known to focus to two equilibrium regions centered at the long faces of the channels. (b) The particle shapes, stretching ratios, and ellipsoid dimensions evaluated in the current work. (c) The microfluidic device (upper drawing) used for shape-based separation consists of a simple straight 4-cm long channel, with $W=25,30$, or $35 \mu \mathrm{m}$, and $H=47 \mu \mathrm{m}$. At the inlet (middle drawing, left), particles are initially randomly distributed within the fluid. Equilibrium positions $\left(X_{\text {eq }}\right)$ are measured at the channel outlet (middle drawing, right), $4 \mathrm{~cm}$ downstream of the inlet, where particles are assumed to be inertially focused due to the combined effect of $F_{\mathrm{L}_{\mathrm{w}}}$ (wall-effect lift) and $F_{\mathrm{L}_{\mathrm{s}}}$ (shear-gradient lift). Overlaid pictures (scale bar $=$ $10 \mu \mathrm{m})$ illustrate particle distribution, respectively, at the inlet (right) and outlet (left). The lower images are multiple overlays of frames captured at the channel inlet (left) and outlet (right).

nonspherical particles to uniform locations [41], illustrating that the effect of particle shape on its focusing position is an important parameter to be further investigated. However, it is not clear how particles of controlled aspect rations with 
the same volume would be have under these conditions and whether inertia can be used to sort these particles.

Here we evaluate how the shape of a particle, while conserving volume, modifies its motion and its dynamic equilibrium position in a microchannel under inertial conditions and use these differences to perform shape-based separations. Rod-like particles migrate to a stable position closer to the channel center line than spherical particles with the same volume and align such that they periodically tumble, rotating around a short axis following Jeffery orbits [44], and are pushed away from the channel wall. Numerical simulations and experimental results both yield focusing positions that are dependent on the particle's largest cross-sectional dimension, and simulations indicate particle rotation is necessary for this behavior. From these shape-based differences in focusing positions, we demonstrate passive and high-throughput separation using a particle's largest cross-sectional dimension as a distinguishing marker, independent of the smallest dimension of the particle. We apply this separation to the efficient and highthroughput sorting of budding yeast in view of cell-cycle synchronization (at rates of $60 \mu \mathrm{L} / \mathrm{min}$ or 1500 cells/s compared to 100 cells/s in previous work [32]). The details of the particles and of the design of the devices and experimental methods appear in the Appendix.

\section{RESULTS AND DISCUSSION}

\section{A. Particle motion in microchannels depends on shape}

In Stokes flow, the linearity of the equations of motion leads to the absence of lift forces on particles with sufficient rotational symmetry (such as the ellipsoids investigated here) [42]. Therefore, one must rely on the effects of inertia [28], particle deformability [40], or the non-Newtonian nature of the fluid itself [43] to establish shape-dependent, lift-induced positions of equilibrium within a microchannel. For common applications that are concerned with rigid particles in a Newtonian fluid such as water, one relies on inertia alone.

With finite inertia, ellipsoid particles migrate to inertialfocusing positions within microchannels and undergo different modes of rotational motion. We classify the rotational configurations qualitatively as in-plane rotation when the particle rotates around the y axis, out-of-plane rotation when the rotation contains components around the $\mathrm{x}$ axis, and no rotation when the particle does not rotate while moving downstream [Fig. 2(a)]. At low particle Reynolds numbers $\left(R e_{\mathrm{p}}\right.$, calculated for a sphere with the same volume), because the particles are not focused, their rotation is random; rather than a pure in-plane-rotation, out- of-plane-rotation, or no-rotation mode, they exhibit a mix of rotational behaviors. If the particle rotates in any manner other than a pure in-plane mode, its rotation is classified as out of plane. At $R e_{\mathrm{p}}$ of $0.3,52 \%$ of particles exhibit some out-of-plane rotational behavior, compared to $39 \%$ that exhibit pure in-plane rotation. As the flow rate and $R e_{\mathrm{p}}$ increase from $0.3(Q=20 \mu \mathrm{L} / \mathrm{min})$ to 0.75 $(Q=50 \mu \mathrm{L} / \mathrm{min})$, the percentage of in-plane rotation increases, with a corresponding reduction in the other two modes. These differences can be explained: At higher $R e_{\mathrm{p}}$, more complete migration to the dominant focusing positions on the long edges of a rectangular microchannel is expected. Along these edges, particles rotate around the axis of highest vorticity, perpendicular to this long face,
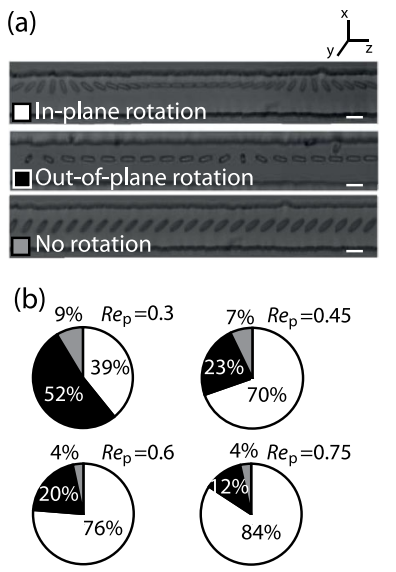

(c)
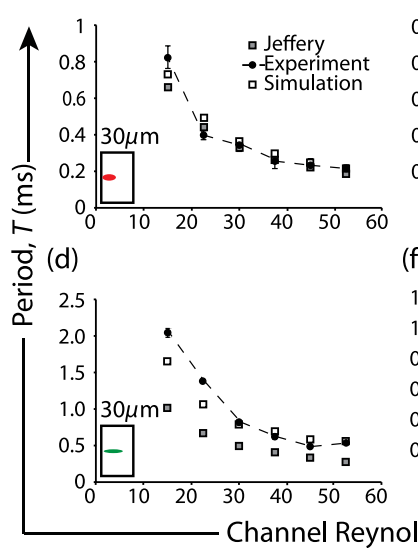

(e)
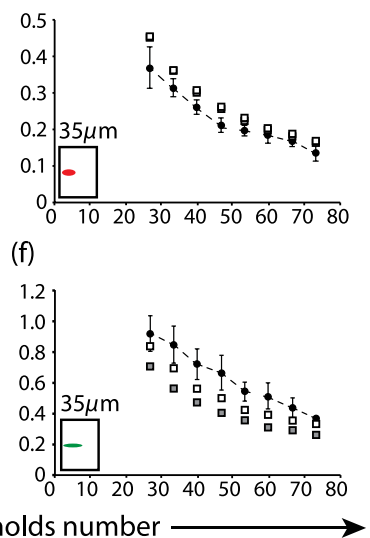

(g)

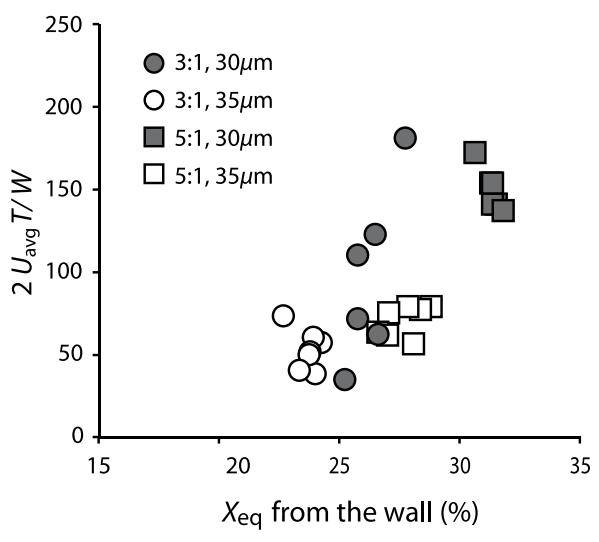

FIG. 2. Rotational motion of ellipsoid particles in a microchannel. (a) Three modes of motion of 1:5 rods are observed in a channel with aspect ratio $\left(A R_{\mathrm{c}}\right)$ of 0.74 : in-plane rotation, out-of-plane rotation, and no rotation. (b) As $R e_{\mathrm{p}}$ (calculated for a sphere of the same volume) is increased from 0.3 to 0.75 , the frequencies of out-of-plane-rotation and no-rotation modes decrease, and most of the particles rotate in-plane around the vorticity axis [color legend shown in (a)]. The periods of rotation from our simulation and the Jeffery formula are plotted along with the experimental results for (c),(e) 1:3 and (d),(e) 1:5 rods in (c),(d) $A R_{\mathrm{c}}=0.64$ and (e), (f) $A R_{\mathrm{c}}=0.74$. Scale bars: $20 \mu \mathrm{m}$. (g) The period of rotation $T$, normalized by average shear rate, increases as the particles get closer to the channel center line due to either an increase in particle length or a decrease in channel width. The normalized value of period is calculated by $T^{*} U_{\text {avg }} /(W / 2)$, where $T$ is the period of rotation, $U_{\text {avg }}$ is the average fluid velocity, and $W$ is the channel width. 
leading to a more uniform in-plane rotational motion. For these well-focused particles, the uniform behavior for rotation is a tumbling motion, or periodic flipping around the long axis that is not rotationally symmetric. Rotational motion of ellipsoidal particles in flow has been previously observed by Jeffery and others [44-46]. Unlike our results, Jeffery did not predict a single axis of rotation but an infinite set of stable orbital motions that depend on initial conditions. However, addition of a little inertia for the fluid or particle has been suggested to lead to the precession of particle orbits to a stable rotational axis in a shear (or parabolic flow) [47,48]. Our results confirm these predictions through the convergence of rotational modes to a single in-plane mode when one increases $R e_{\mathrm{p}}$ from 0.3 to 0.75 .

The period of rotational motion, $T$, is dependent on particle shape. Jeffery demonstrated that an isolated inertialess ellipsoid in the field of an unbounded linear shear flow undergoes an infinite set of periodic closed orbits around the vorticity axis [44]. The orbit period, $T_{\text {orbit, }}$ of an ellipsoid with aspect ratio $A R$ is given by

$$
T_{\text {orbit }}=\frac{2 \pi}{\gamma}\left(A R+\frac{1}{A R}\right)
$$

where $\gamma$ is the local shear rate. The experimentally observed rotational dynamics of ellipsoidal particles (aspect ratios $A R=1: 3$ and 1:5) in a channel flow follows a similar dependence of the rotational period on the ellipsoid aspect ratio, with an increasing rotational period for longer particles (Fig. 2). This observation suggests that inertia does not modify the behavior notably compared to the Jeffery prediction for Stokes flow.

Since the Jeffery orbit is distinctive, the reproduction of similar trends in simulation is a strong indication that the correct dynamics have been captured. From our simulations (explained in detail in the Appendix), the trajectories of particles (center of mass) at different initial positions converge to an equilibrium position. The trajectories oscillate when particles are close to the wall due to the rotation of the ellipsoidal particles. (See Fig. 1 in Ref. [49].) The period of oscillation predicted by our simulation and the Jeffery formula for particles with aspect ratio 1:3 [Figs. 2(c) and 2(e)] and 1:5 [Figs. 2(d) and 2(f)] in channels with aspect ratios of 0.64 [Figs. 2(c) and 2(d)] and 0.74 [Figs. 2(e) and 2(f)] largely agree with each other and with the experimental results, suggesting only small contributions from finite inertia and the curvature of the velocity field. Note that a larger deviation from the Jeffery predictions is observed for particles with a 5:1 aspect ratio. It is likely that, for these longer particles, the gradient in the velocity in our channels plays a larger role in rotational dynamics. In our system, unlike for Jeffery orbits, the shear rate is not identical throughout the flow; it reverses sign at the channel center line and increases toward the wall. The longer 5:1 particles are exposed to regions of flow reversal (relative to the particle frame of reference) across the channel center line from where focusing occurs. These particles are observed to rotate more slowly (i.e., they have a longer period of rotation) than the Jeffery predictions that rely solely on the local shear rate at the particle center [Fig. 2(g)].

The dominant tumbling rotational motion suggests a mechanism by which particles of differing aspect ratios focus to unique positions within a channel cross section. Because the particle shape is not spherical, when the major axis rotates to the orientation perpendicular to the plane of the wall, wall-effect lift increases substantially due to the closer distance, acting to push the particle away from the wall. When the major axis aligns with the flow, wall-effect lift decreases, and the particle migrates back toward the wall. Relative dominance in the lift away from the wall over that toward the wall integrated over time as the particle tumbles suggests that average equilibrium positions are closer to the center line for higher-aspect-ratio particles. In other words, wall-effect lift away from the wall is strongly dependent on distance from the wall and becomes much stronger than the balancing shear-gradient lift in the near-wall region that acts on rotating ellipsoids.

\section{B. Particle shape influences the location of the steady-state focusing position}

Particles with different shapes have different inertial equilibrium positions. This variation in equilibrium position is seen in histograms of lateral positions of the particles for channels with aspect ratios of $0.53,0.64$, and 0.74 and flow rates in the range from $20-110 \mu \mathrm{L} / \mathrm{min}$. (See Fig. 3 in this paper and Fig. 2 in Ref. [49].) The possibility of particle separation at each condition is better characterized through plotting a parameter related both to differences in equilibrium position and to the variation in this position for the population of particles, which we call the separability factor ( $S F_{\text {Type1,Type2) }}$ [Eq. (1)]. Practically, $S F_{\text {Type1,Type2 }}$ indicates the expected separation performance and is calculated as the difference in average focusing positions $X_{\text {eq }}$ between two particle shapes or "types," $X_{a}$ and $X_{b}$, normalized by the mean of their standard deviations, $S D_{a}$ and $S D_{b}$ (Fig. 3 in Ref. [49]), as follows:

$$
S F_{a, b}=\frac{\left|X_{a}-X_{b}\right|}{\operatorname{mean}\left(S D_{a}, S D_{b}\right)} .
$$

In 35- $\mu$ m-wide channels (channel aspect ratio $A R$ closer to 1 ), at Reynolds numbers higher than 10, inertial effects start to concentrate both spherical and rod-shaped particles [Fig. 3(a)]. Spheres start to accurately focus and occupy four focusing positions, while rods are largely spread along the channel width. As fluid inertia increases ( $R e=72, Q=110 \mu \mathrm{L} / \mathrm{min}$ ), spherical particles migrate to locations closer to the walls when compared to particles that have higher $A R$ [Figs. 3(b) and 3(c)]. In addition, some of the particles are focused to other positions, on the top and bottom faces of the channel. The existence of four 

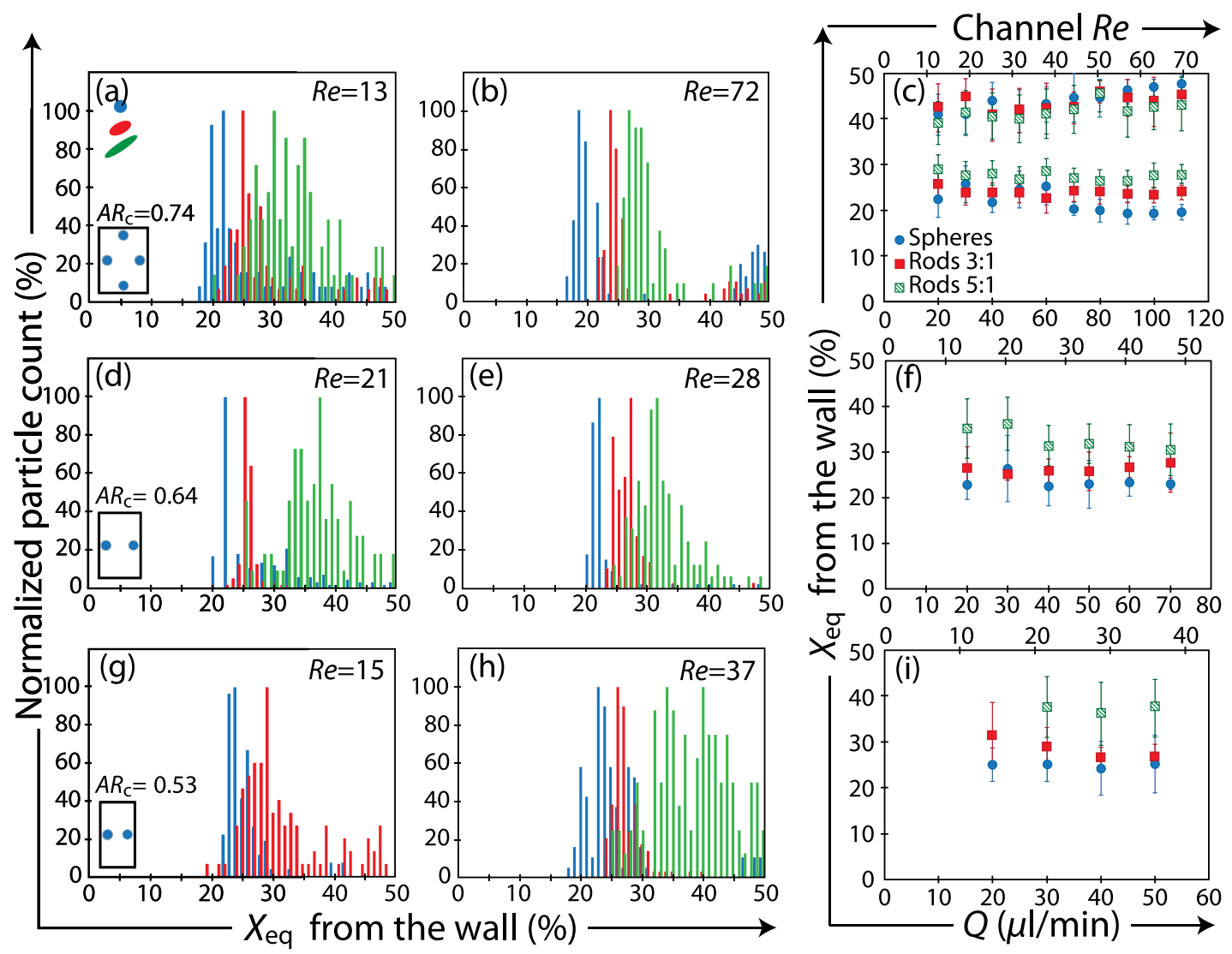

FIG. 3. Focusing distributions depend on particle shape, channel aspect ratio $\left(A R_{\mathrm{c}}\right)$, and the Reynolds number of the flow. (a),(b),(d), (e),(g),(h) Histograms of $X_{\text {eq }}$ for spheres, 1:3 rods, and 1:5 rods, in different channel cross sections and at different flow rates, indicate that equilibrium positions vary for different shapes. (a),(b) $A R_{\mathrm{c}}=0.74$, and $Q=20$ and $110 \mu \mathrm{L} / \mathrm{min}$, respectively; (d), (e) $A R_{\mathrm{c}}=0.64$, and $Q=30$ and $40 \mu \mathrm{L} / \mathrm{min}$, respectively; (g),(h) $A R_{\mathrm{c}}=0.53$, and $Q=20$ and $50 \mu \mathrm{L} / \mathrm{min}$, respectively. (c),(f), (i) Averaged $X_{\text {eq }}$ is plotted for all channel geometries and flow conditions tested, with error bars indicating the standard deviation obtained from at least 100 measurements.

equilibrium positions, expected for such $A R(0.74)$, is problematic for separation applications. Decreasing channel width from 35 to $30 \mu \mathrm{m}$ changes the $A R$ of the channel cross section to 0.64 , which leads to migration to only two distinct equilibrium positions. At $30 \mu \mathrm{L} / \min (R e=21)$, 1:5 rods are initially separated from spheres and 1:3 rods [Fig. $3(\mathrm{~d})] ; \quad S F_{\text {Spheres,Rods1:3 }}=0.24, \quad S F_{\text {Rods1:3,Rods1:5 }}=$ 2.26. (See also Fig. 3 in Ref. [49].) As $Q$ is increased to $40 \mu \mathrm{L} / \min (R e=28)$, both families of rods migrate further away from spheres and from each other [Fig. 3(e)], suggesting the possibility of a better shape-based separation: $S F_{\text {Spheres,Rods1:3 }}=0.85, S F_{\text {Rods1:3,Rods1:5 }}=1.46$. As $R e$ is increased further $(R e=49,70 \mu \mathrm{L} / \min )$, rods tend to move closer to the walls where spheres are located, reducing the gap between focusing positions [Fig. 3(f)]: $S F_{\text {Spheres,Rods1:3 }}=1.05, S F_{\text {Rods1:3,Rods1:5 }}=0.61$. Decreasing channel width further to $25 \mu \mathrm{m}$, aspect ratio 0.53 , makes it difficult to uniquely focus all particles [Fig. 3(g)]. Indeed, even at $R e=37(50 \mu \mathrm{L} / \mathrm{min}), 1: 5$ rods are still not focused to a unique streamline [Figs. 3(h) and 3(i)]. This result arises also partly because, especially with larger rods, this narrow channel is prone to clogging. These results clearly suggest that optimum conditions exist that maximize the distance between particle positions and allow for the most efficient particle separation based on aspect ratio. The experiments also suggest that, above a cutoff $R e$ where particles are already focused, increasing $R e$ further will result in the convergence of focusing positions for all three particles to a position closer to the wall. This observation agrees with previous numerical analysis by Di Carlo et al. [50]. The lift force from a wall-directed shear gradient increases faster with increasing $R e$ than the balancing wall-effect lift, leading to a shift in equilibrium position closer to the wall. Convergence of the focusing positions for normally segregated shaped particles is likely because this effect acts more strongly on particles with initial focusing positions further from the wall.

\section{Spheres with similar rotational diameter predict focusing positions for rod-shaped particles}

Whatever the channel aspect ratio, experimentally determined focusing positions agree best with the hybrid 


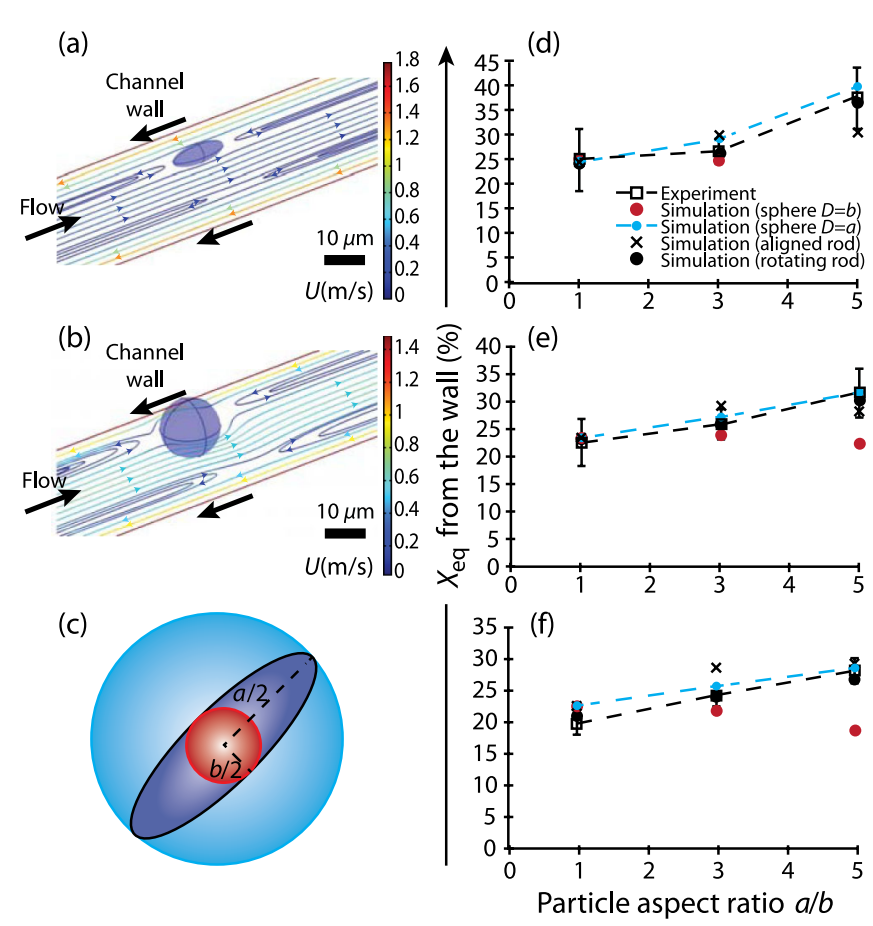

FIG. 4. Simulations allow accurate prediction of focusing positions for shaped particles. Simulation result and streamlines are shown for flow in the reference frame of (a) a focused rod and (b) a spherical particle in a straight channel. (c) Simulations are performed considering particle shape and aspect ratio with (i) the particle rotating (rotating rods) and (ii) the rod constrained from rotation (aligned rods), and also considering spheres with diameters that correspond to the rods' (iii) longest dimension (spheres $D=b$ ) and (iv) smallest dimension (spheres $D=a$ ). (d),(e),(f) Comparison of equilibrium positions away from the wall, obtained by experiments and numerical simulations, for (d) $A R_{\mathrm{c}}=0.53, Q=50 \mu \mathrm{L} / \mathrm{min} ; \quad$ (e) $A R_{\mathrm{c}}=0.64, Q=$ $40 \mu \mathrm{L} / \mathrm{min}$; and (f) $A R_{\mathrm{c}}=0.74, Q=110 \mu \mathrm{L} / \mathrm{min}$.

computational method that allows for simulation of rotating rods. Simulations with aligned rods, which are constrained not to rotate and with the major axis pointed in the flow direction, exhibit considerable differences in comparison with experiments [Fig. 4(a)]; high-aspect-ratio particles occupy equilibrium positions significantly closer to the wall than is experimentally observed. These results further implicate strong lift during rotation as being responsible for shape-dependent differences in focusing. Aligned rods cannot rotate freely following Jeffery orbits in which the rods are periodically pushed away from the wall. Simulations with spheres with diameter corresponding to the minor axis of the particle (spheres $D=b$ ) [Figs. 4(c)-4(f)] do not match the experimental results well, either. However, simulations with spheres with diameters corresponding to the major axis of the particle (spheres $D=a$ ) align much more closely with the experimental results [Figs. 4(b) and 4(d)-4(f)]. This simulation indicates that rod-shaped particles with $180^{\circ}$ rotational symmetry follow the focusing trend of spheres with similar rotational diameter. These results agree with those obtained recently by Hur et al. with other shapes and larger dimensions [41] and provide a simple approach for modeling and predicting the focusing positions for nonspherical particles. Particles without this rotational symmetry are subject to additional noninertial lift forces following the work of Bretherton [42].

\section{Application to shape-based separation}

Using the significant differences in lateral equilibrium position emphasized above, we have conducted labelfree enrichment and collection of shaped particles. Figure 5 illustrates the separation results for several configurations of shape-activated particle-sorting (SAPS) devices, where the experimental parameters (channel aspect ratio and flow rate) have been chosen based on the separability results obtained in the previous sections. (See Fig. 3 in Ref. [49].) To quantify the efficiency of separation, we report three parameters defined in the methods: extraction yield $(E Y)$, extraction purity $(E P)$, and enrichment ratio (ER). (See Figs. 5(d)-5(f).) To better achieve specific separations, we have designed devices with three arrangements of outlet resistances, which provide separate relative-capture ratios of the fluid at different outlets [Figs. 5(a)-5(c)].

By tuning device parameters, we demonstrate a range of possible separations between spheres, 1:3 rods, and 1:5 rods. The SAPS device A, $25 \mu \mathrm{m}$ wide $\left(A R_{\mathrm{c}}=0.53\right)$, with identical fluidic resistance for each outlet, is shown in Fig. 5(a). For this design, most spheres and 1:3 rods exit from outlet 1 , while $1: 5$ rods are predominantly captured from outlet 2 [Fig. 5(d) and the second movie in Ref. [49].] In agreement with $S F$ measurements for these flow conditions (see Fig. 3 in Ref. [49]), 1:5 rods are found to have a high extraction yield in outlets 2 and 4 (86\% of 1:5 rods) with up to $90 \%$ purity, compared to the results for $1: 3$ rods and spheres, which are mainly collected together in outlets 1 and 5 (83\% of all spheres and $70 \%$ of all 1:3 rods injected) [Figs. 5(g) and 5(j)]. To investigate another scenario of separation and with a higher flow rate, we tune the experimental conditions to a channel aspect ratio of 0.64 , and a flow rate of $80 \mu \mathrm{L} / \mathrm{min}$ [SAPS device B, Fig. 5(b)], and we modify the ratio of fluidic resistance between the different outlets $\left(\alpha_{1: 2}=3 / 4, \alpha_{1: 3}=1 / 2\right)$. Contrary to the previous results, we achieve excellent extraction yield for spherical particles (with $85 \%$ of all spheres being found in outlets 1 and 5), while both types of rods are collected together (with $90 \%$ of all rods being extracted in outlets 2 and 4), leading to an extraction purity of $96 \%$ for spheres. [See Figs. 5(e), 5(h), and 5(k) and the first movie in Ref. [49].] These results are still in agreement with $S F$ values. Decreasing the flow rate to $60 \mu \mathrm{L} / \mathrm{min}$ in a $30-\mu \mathrm{m}$ channel with $A R_{\mathrm{c}}=0.64$ allows us to separate all three types of particles while slightly decreasing the purity of spheres, as predicted by $S F$ measurements. The presence of seven 
(a)
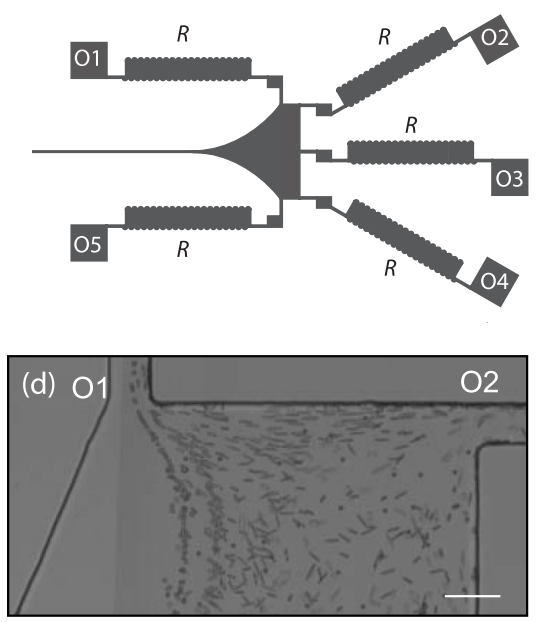

(g) $\square$ Spheres $\square$ Rods 3:1 $\square$ Rods 5:1

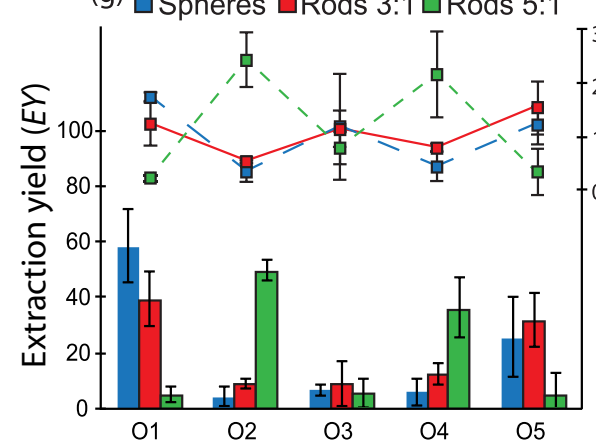

(j)

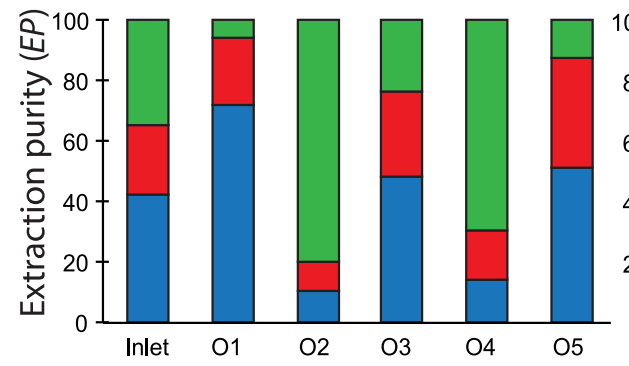

(b)
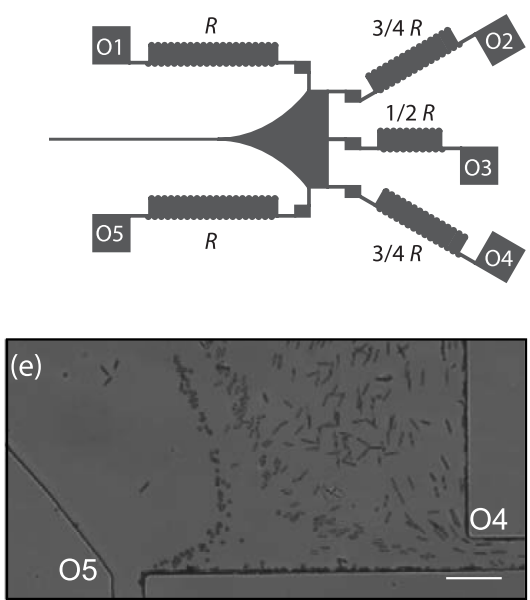

(h) (c)
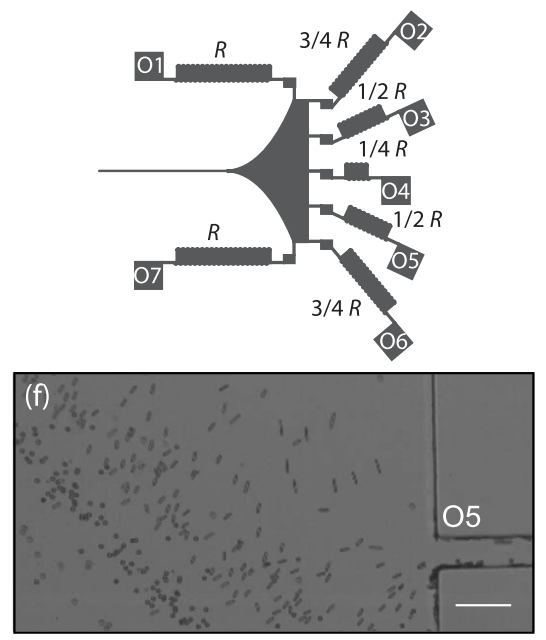

(i)

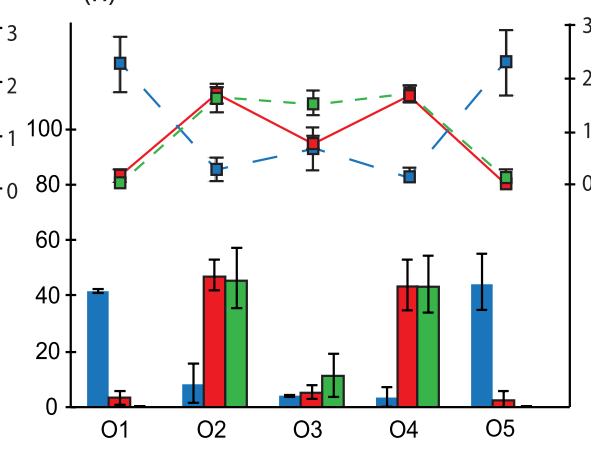

(k)

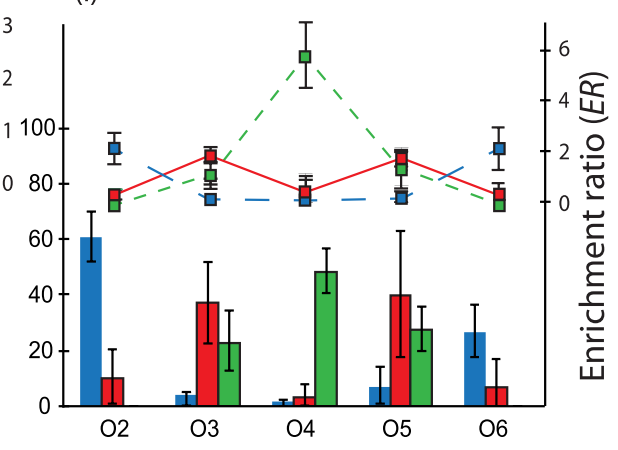

(I)

FIG. 5. Separation results for 6- $\mu \mathrm{m}$ spherical and ellipsoid particles. The collected ratio of particles is shown at each outlet, considering the extraction yield $E Y$ and the enrichment ratio $E R(\mathrm{~g}),(\mathrm{h}),(\mathrm{i})$, and the extraction purity $E P(\mathrm{j}),(\mathrm{k}),(\mathrm{l})$. Each value shows the mean \pm SD from three independent experiments. Three configurations of the SAPS device are considered (a),(b),(c), and stacked pictures of the separation are shown for each configuration (d),(e),(f). Scale bar: $50 \mu \mathrm{m}$. (a) $A R_{\mathrm{c}}=0.53, Q=40 \mu \mathrm{L} / \mathrm{min}$, five outlets with equal resistances (R); (b) $A R_{\mathrm{c}}=0.64, Q=80 \mu \mathrm{L} / \mathrm{min}$, five outlets (O1 to O5) with $\alpha_{1: 2}=3 / 4$ and $\alpha_{1: 3}=1 / 2$; (c) $A R_{\mathrm{c}}=0.64, Q=70 \mu \mathrm{L} / \mathrm{min}$, seven outlets (O1 to O7) with $\alpha_{1: 2}=3 / 4, \alpha_{1: 3}=1 / 2, \alpha_{1: 4}=1 / 4$. In (i) and (1), since no particles were captured from outlets $\mathrm{O} 1$ and $\mathrm{O} 7$, only the collected ratio of particles at outlets $\mathrm{O} 2$ to $\mathrm{O} 6$ appear in the graphs.

outlets in SAPS device C [Fig. 5(c)] provides a more accurate separation between streamlines (SAPS device C: $\alpha_{1: 2}=$ $\left.3 / 4, \alpha_{1: 3}=1 / 2, \alpha_{1: 4}=1 / 4\right)$. Indeed, we have isolated $88 \%$ of spheres in outlets 2 and 6 with $87 \%$ purity, $49 \%$ of $1: 5$ rods in outlet 4 with $78 \%$ purity, and, more interestingly, $77 \%$ of $1: 3$ rods with $80 \%$ purity [Figs. 5(f), 5(i), and 5(1)].

We have also examined and confirmed the possibility of separating 3- $\mu \mathrm{m}$ spheres and 3- $\mu \mathrm{m}$ derived ellipsoids with three different aspect ratios by applying the same concept as has been used for separating 6- $\mu \mathrm{m}$ beads with slightly modified parameters. Using SAPS device D (Fig. 4.A in Ref. [49]), we collected spheres in outlets 1 and 5 with $90 \%$ yield (EY) and up to $90 \%$ purity (EP), as well as $81 \%$ yield of $1: 3$ rods in outlets 2,3 , and 4 , and $97 \%$ yield of $1: 5$ rods in outlets 2, 3, and 4, with up to $88 \%$ purity (EP) of the two types of rods (Figs. 4.B-4.D and the third movie in Ref. [49]). 


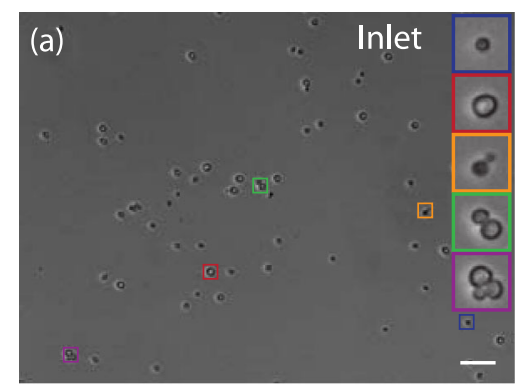

(c)

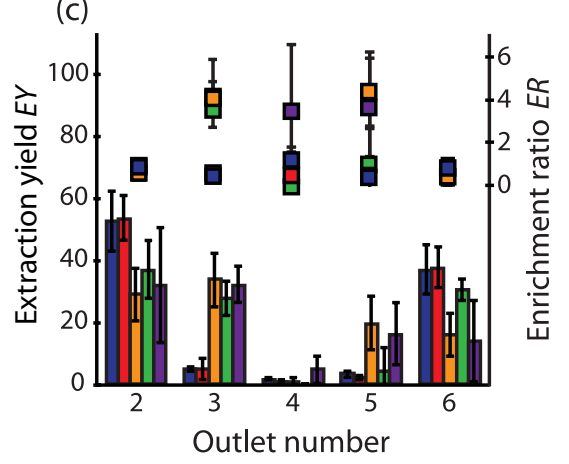

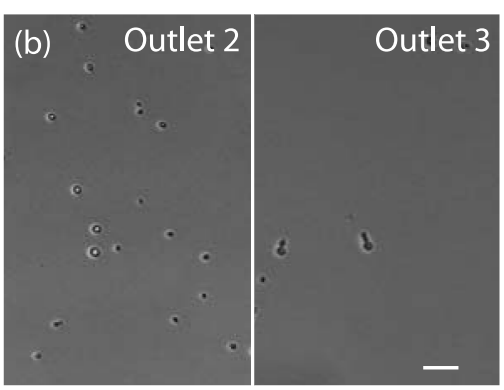

(d)

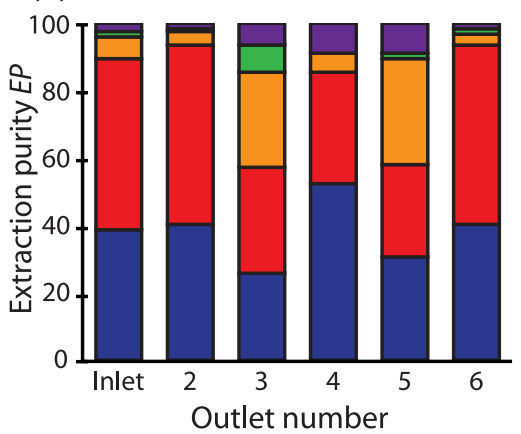

FIG. 6. Yeast-cell sorting in SAPS device C $\left(A R_{\mathrm{c}}=0.64\right.$, seven outlets with $\left.\alpha_{1: 2}=3 / 4, \alpha_{1: 3}=1 / 2, \alpha_{1: 4}=1 / 4\right)$ at $60 \mu \mathrm{L} / \mathrm{min}$. (a) A picture of the cells in the inlet. Cells are categorized into five groups: small single (blue), large single (red), budded (orange), doublet (green), and aggregate (purple). (b) Singles have a high extraction yield in outlet 2, while in outlet 3, the purity of budded cells increases. (c) The collected ratio of particles is shown at each outlet, considering the extraction yield $E Y$ and enrichment ratio ER and (d) extraction purity $E P$. Each value shows the mean \pm SD from three independent experiments. Since no particles were captured from outlets $\mathrm{O} 1$ and $\mathrm{O} 7$, only the collected ratio of particles at outlets $\mathrm{O} 2$ to O6 appear in the graphs.

\section{E. Application to passive and high-throughput yeast-cell sorting}

Shape-based separation using inertial effects allows yeastcell sorting and cell-cycle synchronization. Understanding of the cell cycle is the subject of current research, which is often explored using yeast cells (Saccharomyces cerevisiae) because of its well-known genetics and characteristic shape changes: Budding yeast cells elongate from a sphere to a bispherical twin or a larger aggregate [2,51]. To understand gene-expression profiles specific to each of these stages, it is necessary to synchronize the yeastcell cycle. This synchronization at given cycle stages is generally done by using (i) chemicals (metabolic agents) that disturb the cell physiology, (ii) a temperature increase, or (iii) size-based elutriation, which isolates the smaller cells [52]. The first methods interfere with the cell metabolism, while elutriation only provides young cells not yet in active division [53]. Thus, there remains a need for a noninvasive and drug-free, continuous method for shapebased yeast-cell sorting and synchronization [32].

Using the SAPS device $\mathrm{C}$ previously described $\left(A R_{\mathrm{c}}=0.64\right.$, seven outlets with $\alpha_{1: 2}=3 / 4, \alpha_{1: 3}=$ $\left.1 / 2, \alpha_{1: 4}=1 / 4\right)$, we have conducted yeast sorting at various flow rates. Figure 6 summarizes the separation results obtained at $60 \mu \mathrm{L} / \mathrm{min}$, with pictures showing inlet and outlet contents [Figs. 6(a) and 6(b)]. Nondividing singles are found to have a high extraction yield in outlets 2 and 6 (with $90 \%$ of small singles and $91 \%$ of large singles being recovered in these outlets), with up to $94 \%$ purity, while budded yeast cells are mainly collected in outlets 3 and 5 (with $54 \%$ of budded yeast being recovered, with up to $31 \%$ purity, compared to $6.6 \%$ purity at the inlet) [Figs. 6(c) and $6(\mathrm{~d})]$. The higher throughput of our SAPS device $(60 \mu \mathrm{L} / \mathrm{min}$, or 1500 cells/s compared to 100 cells/s in previous work [32]) could be further increased an order of magnitude by increasing cell concentration and by parallelization of the focusing channels, as we have demonstrated previously in our laboratory [37,38]. The levels of purity and enrichment especially needed for this synchronization application could be improved by cascading devices in series.

\section{CONCLUSION}

We have demonstrated that the inertial separation of particles with different shapes is possible and tunable with channel geometry and flow conditions. This work is the first of its kind to demonstrate a practical solution to continuously filtering particles that have similar dimensions along one axis but that vary along another axiswhich cannot effectively be accomplished with traditional filtration methods. The approach is passive, simple, and continuous, using only inertial effects present in simple 
straight channels. Other advantages are (i) the absence of any external setup to induce particle separation, as opposed to active methods such as DEP, and (ii) increased processing rates compared to DLD and HDF techniques, which require particularly low flow rates $(60 \mathrm{~nL} / \mathrm{min}$ [30] and 2-3 $\mu \mathrm{L} / \min$ [31] vs our $40-80 \mu \mathrm{L} / \mathrm{min}$ ), since the underlying separation force does not increase with flow rate in previous techniques while it does with inertial separation.

The predictability of inertial focusing of nonspherical particles is of interest to various research areas. There are many arbitrarily shaped particles in biology and industrial processing that are counted and analyzed. As an example, the deviation from spherical symmetry has been recently demonstrated to result in a considerable increase in cytometry uncertainty [54]. The precise alignment of shaped particles by inertial focusing, and especially the predictability of this alignment, would help to address this kind of uncertainty and to produce more reliable measurements [55]. One potential application is the fluidic alignment of barcoded particles. Barcoded particles, which are fabricated using approaches such as stop-flow lithography, are used for multiplexed and high-throughput biochemical assays. These particles are usually aligned by sheath flow [7,56], which can lead to unstable focusing, or by active guiding rails [57], which complicate their integration into microsystems. Inertial effects may enable precise control of the alignment and focusing of barcoded particles for the optical reading of their patterns. Similarly, our results suggest design criteria, because, although particles rotate in our channels, maintaining particles in a relatively fixed orientation for the longer period needed for reading can be achieved by increasing the particle aspect ratio. Another application is the sorting of microalgae prior to cytometry, as phytoplankton possesses a large variety of shapes and sizes $[5,6]$. Nonspherical objects rotate and translate vertically in an oscillatory pattern in the channel, and, depending on their initial angle, these organisms with the same length may pass through the interrogation region at different angles, causing different scatter signals. Shape-based separation prior to interrogation would allow a more effective identification of marine microorganisms in water.

More interestingly, inertial shape-based separation is possible for a large range of particle sizes. The differential focusing of larger nonspherical particles (up to $100 \mu \mathrm{m}$ ) has been shown previously [41]. Focusing of particles as small as $2 \mu \mathrm{m}$ has also been demonstrated in our lab [27], while particles smaller than $2 \mu \mathrm{m}$ require higher flow rates and pressures, necessitating materials with higher bond strengths, such as Thermoset Polyester (TPE) [58]. The possibility of separating $2-3-\mu \mathrm{m}$ spheres and rods as demonstrated here opens a new range of applications in separating bacteria to synchronize populations at different stages of cell growth. Depending on the stage of the cell cycle, rodlike bacteria (e.g., bacilli) can as much as double their length while maintaining the same short dimension. Enrichment of these bacteria by life-cycle stage can avoid cell-cycle-dependent noise and help microbiologists in synchronizing a population to better understand population dynamics, environmental effects leading to desynchronization, and stochasticity in single-cell behavior [59]. New applications may also be targeted outside of biotechnology, e.g., in separation of cement microparticles into pure fractions, since cement strength and stability are critically linked to particle shape and size. A way to filter highly defined particles without clog-prone filters would aid in the development of optimized cement formulations-saving material costs for various construction applications.

\section{ACKNOWLEDGMENTS}

M.M. and E.S. contributed equally to this work. We thank Dr. Soojung C. Hur for her helpful advice concerning the design of device geometry, especially for particle separation. This work is partially supported by NSF Grant No. 0930501.

\section{APPENDIX}

\section{Microfluidic device fabrication and geometry}

Microfluidic devices have been fabricated using common polydimethylsiloxane (PDMS) replica-molding processes [58]. Briefly, standard lithographic techniques are used to produce a mold from a silicon master that is spincoated with SU-8 photoresist. PDMS chips are produced from this mold using the Sylgard 184 Elastomer Kit (Dow Corning Corporation) and a cross-linker-to-polymer ratio of 1:10. To enclose the channels, PDMS and glass are both activated by air plasma (Plasma Cleaner, Harrick Plasma, $500 \mathrm{mTorr}, 30 \mathrm{sec}$ ) before being bonded together. Our primary device is a straight rectangular channel, $4 \mathrm{~cm}$ in length (a distance expected to be long enough for particles to be laterally focused in their dynamic equilibrium positions $\left(X_{\text {eq }}\right)$ [35]), and with a cross section of $47 \mu \mathrm{m}$ deep by 25,30 , or $35 \mu \mathrm{m}$ wide. (Channel aspect ratios $A R_{\mathrm{c}}$ are therefore $0.53,0.64$, and 0.74 , respectively.)

For particle separation, our SAPS device is composed of (i) the previously described straight focusing channel, (ii) a gradually expanding region downstream of the focusing channel, and (iii) five or seven branched outlets with tuned fluidic resistors [Figs. 6(a)-6(c)]. Indeed, as previously reported by Hur et al. [40], a gradually expanding region maintains focused particles in the focusing streamline while enhancing their $X_{\text {eq }}$ differences when compared to straight-angled expansions. The tuned fluid resistors in the form of serpentine channels minimize the flow-ratio distortion caused by any small variation in the outlet fluidic resistance (such as small variation in tubing length or small debris) [40]. These serpentine channels also offer the ability to precisely configure each outlet by tuning the ratio of outlet fluidic resistances to collect more or less of a given 
stream. $\alpha$ represents this ratio of outlet flow rates $\left(\alpha_{1: 2}=\right.$ $\left.Q_{\mathrm{O} 1} / Q_{\mathrm{O} 2}\right)$, which also represents the ratio of outlet fluidic resistances. For both geometries, filters located at the inlet prevent the channel from being clogged by aggregates.

\section{Bead fabrication and injection}

Spherical beads of 3 and $6 \mu \mathrm{m}$ (Polyscience) are stretched into rods with different aspect ratios $(R=1: 3$ and 1:5) (Fig. 1), following the protocol published by Champion et al. [60]. Spherical polystyrene particles (beads) are suspended in a solution of $75^{\circ} \mathrm{C}$ water and hot-water-soluble polyvinyl alcohol (PVA) to a final concentration of $10 \% \mathrm{wt} / \mathrm{vol}, 5 \% \mathrm{wt} / \mathrm{vol}$ glycerol, and $0.08 \% \mathrm{wt} / \mathrm{vol}$ beads. This solution is spread and dried overnight on a $19 \times 27-\mathrm{cm}$ flat surface. The films are then stretched in mineral oil at $120^{\circ} \mathrm{C}$ on a custom-made apparatus and dried at room temperature for 20 minutes. To recover the rod-shaped particles, the films are washed with isopropanol and dissolved in a 30\% isopropanol-water solution at $75{ }^{\circ} \mathrm{C}$. The particles are finally washed 8 times, each time with decreasing amounts of isopropanol, in order to remove all PVA from the particle surface. Particle suspensions are injected into tested devices, at a maximum concentration of $1 \times 10^{6}$ beads $/ \mathrm{mL}$, using a syringe pump (Harvard Apparatus PHD 2000) and a glass syringe (Hamilton), at flow rates $Q$ ranging from $20-110 \mu \mathrm{L} / \mathrm{min}$.

\section{High-speed imaging for study of inertial focusing and particle motion}

Image sequences have been recorded $4 \mathrm{~cm}$ away from the inlet using a Phantom v7.3 high-speed camera and the Phantom Camera Control Software (Vision Research, Inc.). Based on these images and using a MATLAB custom-built code, the size and center of individual particles have been determined in each image frame to estimate the average particle equilibrium position $X_{\text {eq }}$. Particle motion in channels with 0.64 and 0.74 aspect ratios has also been studied through high-speed images, to determine and characterize the modes of particle rotation.

The average particle equilibrium position $X_{\mathrm{eq}}$ is calculated by measuring the distance between the particle center and the channel wall. $X_{\text {eq }}$ equal to $0 \%$ or $50 \%$ indicates that the particle center is located at the channel wall or the channel center, respectively. To obtain statistical significance, more than 100 data points have been measured for each particle type and flow condition, with less than $1 \%$ error for each point caused by the resolution of the camera. The separability factor $S F_{\text {Type1,Type2 }}$ has been calculated as the difference in $X_{\text {eq }}$ between two particle shapes, normalized by the average of their standard deviations (Fig. 3 in Ref. [49] and Eq. (1) in this paper), and this factor indicated the expected separation performance.

By analyzing high-speed images, we have determined the dominant modes of rotation at the end of the channel
[Fig. 2(a)]. The observation frequency of each of these modes for the channel with $A R_{\mathrm{c}}=0.74$ is reported at different flow rates [Fig. 2(b)]. The period of the rotation $T(\mu \mathrm{s})$ is calculated by counting the number of frames required for a particle to make a half rotation. The number of frames for a half-period is converted to microseconds simply by using the frame rate of the image sequence and then multiplying by 2 to give the period of a full rotation.

\section{Simulations}

Two methods of simulation have been used to calculate equilibrium positions for shaped particles.

(1) To simulate steady-state focusing positions, we use a numerical model that solves the 3D incompressible Navier-Stokes equations in the reference frame of a spinning particle $[50,61]$. For these simulations, while the position of the particle is fixed, the dynamics of the system are updated to achieve force- and torque-free conditions for the particle (i.e., the steady-state behavior). Using this method, we conduct a series of simulations for various particle positions along the short axis of the channel (at $z=0$ ), which yield the steady-state lateral force for a particle held to each particular position. Because the rotation rate of the particle is updated as a slip condition on the particle surface, only particles that are rotationally symmetric about the vorticity axis of the flow (e.g., spherical particles, and ellipsoids rotating around their rotationally symmetric axis) can be accurately simulated. Therefore, for ellipsoid particles with 1:3- and 1:5-dimension ratios that are experimentally observed to prefer a tumbling motion, we can simulate only horizontally aligned positions with no rotation. For both spherical and ellipsoid particles, the position where the lateral force curve crosses zero is interpolated to find the dynamic equilibrium position. Simulations for nonspherical particles (aspect ratios 1:3 and 1:5) are conducted (i) assuming that particles are rods but do not rotate in the channel (aligned rods), (ii) assuming that particles are spheres with their small dimension $(b)$ as the diameter of the sphere (spheres $D=b$ ) or (iii) assuming that particles are spheres with their large dimension $(a)$ as the diameter of the sphere (spheres $D=a$ ).

(2) To predict the particle focusing position of nonspherical particles for the more accurate case of (iv) taking their rotation into account (rotating rods) and to simulate the dynamics and direction of particle rotation, we use a fully coupled hybrid computational approach that integrates the lattice Boltzmann model for the dynamics of the fluid and the lattice spring model for the motion of rigid ellipsoidal particles [62]. The lattice Boltzmann model is a lattice-based numerical method for 
simulating hydrodynamic flows governed by the incompressible Navier-Stokes equations [63,64]. The flow is modeled by simulating the time evolution of a density distribution function, $f_{i}(\mathbf{r}, t)$, that describes the mass density of "fluid particles" with velocity $\mathbf{c}_{i}$ at a lattice node $\mathbf{r}$ at time $t$. We use a three-dimensional D3Q19 model with 19 velocities [63]. The time evolution of the distribution function is governed by the discretized Boltzmann equation: $f_{i}\left(\mathbf{r}+\mathbf{c}_{i} \Delta t, t+\Delta t\right)=f_{i}(\mathbf{r}, t)+\Omega[f(\mathbf{r}, t)] . \quad$ The collision operator $\Omega[f(\mathbf{r}, t)]$ accounts for the change in $f_{i}$ due to instantaneous collisions at the lattice nodes. The flow parameters are calculated as follows: The fluid density $\rho=\Sigma_{i} f_{i}$, the momentum $\mathbf{j}=\rho \mathbf{u}=\Sigma_{i} f_{i} \mathbf{c}_{i}$, where $\mathbf{u}$ is the macroscopic fluid velocity, and the stresses $\Pi=\sum_{i} f_{i} \mathbf{c}_{i} \mathbf{c}_{i}$. The solid particles are modeled by a set of lattice nodes located on the outer particle surface and connected by rigid bonds [20]. In this representation, we simulate particles as thin solid shells filled with a viscous fluid. We have verified that, for a low-frequency particle rotation observed in our simulations, the viscous fluid inside particles behaves as a solid and the particles follow the rigid-body dynamics. Thus, the total particle mass combines the contributions due to the shell and the internal fluid [64]. To capture the dynamics of the particle motion, we calculate the total force and torque on the solid particles due to the fluid and find the translational and rotational displacement of the particle by integrating Newton's equation of motions. We then update the positions of lattice nodes on the particle surface.

The lattice models for fluid and solid are coupled through appropriate boundary conditions. Specifically, we use an interpolated bounceback scheme at the moving solid-fluid interface that provides second-order accuracy $[62,65]$. We have previously validated our hybrid model in the limit of low Reynolds numbers and used it to examine microchannel flows with rigid and compliant particles [20-24,66,67]. In order to compare the simulations with experimental results, we set the dimensions of channel, particle size, and Reynolds number equal to the corresponding experimental parameters. At the beginning of the simulations, the particles are released at different positions and their trajectories are monitored. For a given set of flow parameters, particles released at different initial positions migrate to a common equilibrium trajectory. The equilibrium trajectory exhibits oscillations due to the periodic rotation of particles. By averaging the trajectories over one oscillation period, we find the mean equilibrium positions of particles.

The equilibrium orientations of the particles have also been predicted through the experiments [49].

\section{Experiments and analysis for shape-based separation of beads}

For separation experiments, a mixture of spheres and rods of 1:3 and 1:5 aspect ratios are prepared and injected into a SAPS device to evaluate the separation of different particles from each other. Several flow rates and fluidic resistances have been tested to maximize the separation, and the fractions of different particles collected from each outlet for each of these tested conditions have been measured. Separation can be characterized using three parameters, defined below for a particle type $a$ and an outlet $i$ [Eq. (A1)]. The extraction yield (EY) is determined as the number of particle $a$ extracted in the outlet $i$, over the total number of this particle type injected; $E Y$ reports the outlet collection efficiency of a given particle type. The extraction purity $(E P)$ considers the proportion of particle type $a$ in outlet $i$, relative to the total number of beads extracted in this outlet; $E P$ reports the particle composition of a given outlet. The enrichment ratio $(E R)$ is defined as the proportion of particle $a$ in outlet $i$ to its proportion at the inlet.

$$
\begin{aligned}
& E Y=\frac{N_{a}\left(\text { outlet }_{i}\right)}{N_{a}(\text { inlet })}, \quad E P=\frac{N_{a}\left(\text { outlet }_{i}\right)}{N_{\text {tot }}\left(\text { outlet }_{i}\right)}, \\
& E R=\frac{N_{a}\left(\text { outlet }_{i}\right) / N_{\text {tot }}\left(\text { outlet }_{i}\right)}{N_{a}(\text { inlet }) / N_{\text {tot }}(\text { inlet })} .
\end{aligned}
$$

\section{Application to shape-based separation of yeast cells}

Yeast is cultured in tryptic soy broth (TSB) culture in an incubated shaker $\left(37^{\circ} \mathrm{C}\right)$ for $1 \mathrm{~d}$ prior to the separation experiment. The cultured suspension is diluted in phosphate-buffered saline at a nonlimiting concentration of $1.5 \times 10^{6}$ cells $/ \mathrm{mL}$ and then, similar to the treatment of beads, is injected at various flow rates using a Harvard Apparatus syringe pump and Hamilton glass syringe. The separation behavior is captured through high-speed imaging, with the content of each outlet being analyzed by immediate counting with a Quick-Read hemocytometer. The morphologies of yeast cells are observed and categorized, depending on their cycle state, into (i) small nondividing singles, (ii) large singles, (iii) budded yeast, (iv) doublets, and (v) aggregates that are composed of three cells or more.

[1] S. Mitragotri and J. Lahann, Physical Approaches to Biomaterial Design, Nature Mater. 8, 15 (2009).

[2] S. Martin, Geometric Control of the Cell Cycle, Cell Cycle 8, 3643 (2009).

[3] C. Wongsrichanalai, M.J. Barcus, S. Muth, A. Sutamihardja, and W.H. Wernsdorfer, A Review of Malaria Diagnostic Tools: Microscopy and Rapid Diagnostic Test (RDT), Am. J. Trop. Med. Hyg. 77, 119 
(2007) [http://www.ajtmh.org/content/77/6_Suppl/119 .abstract].

[4] M. Piagnerelli, K. Zouaoui Boudjeltia, D. Brohee, A. Vereerstraeten, P. Piro, J.-L. Vincent, and M. Vanhaeverbeek, Assessment of Erythrocyte Shape by Flow Cytometry Techniques, Journal of Clinical Pathology 60, 549 (2006).

[5] N. Hashemi, J. S. Erickson, J. P. Golden, and F. S. Ligler, Optofluidic Characterization of Marine Algae Using a Microflow Cytometer, Biomicrofluidics 5, 032009 (2011).

[6] N. Hashemi, J. S. Erickson, J. P. Golden, K. M. Jackson, and F. S. Ligler, Microflow Cytometer for Optical Analysis of Phytoplankton, Biosens. Bioelectron. 26, 4263 (2011).

[7] D. C. Pregibon, M. Toner, and P. S. Doyle, Multifunctional Encoded Particles for High-Throughput Biomolecule Analysis, Science 315, 1393 (2007).

[8] S. E. Chung, W. Park, S. Shin, S. A. Lee, and S. Kwon, Guided and Fluidic Self-Assembly of Microstructures Using Railed Microfluidic Channels, Nature Mater. 7, 581 (2008).

[9] D. R. Gossett, W. M. Weaver, A. J. Mach, S.C. Hur, H. T. K. Tse, W. Lee, H. Amini, and D. Di Carlo, Label-Free Cell Separation and Sorting in Microfluidic Systems, Anal. Bioanal. Chem. 397, 3249 (2010).

[10] M. Kersaudy-Kerhoas, R. Dhariwal, and M. P. Desmulliez, Recent Advances in Microparticle Continuous Separation, IET NanoBiotechnology 2, 1 (2008).

[11] S. Choi and J.K. Park, Microfluidic System for Dielectrophoretic Separation on a Trapezoidal Electrode Array, Lab Chip 5, 1161 (2005).

[12] D. W. Inglis, R. Riehn, R. H. Austin, and J.C. Sturm, Continuous Microfluidic Immunomagnetic Cell Separation, Appl. Phys. Lett. 85, 5093 (2004).

[13] N. Pamme, Magnetism and Microfluidics, Lab Chip 6, 24 (2006).

[14] M.P. MacDonald, G. C. Spalding, and K. Dholokia, Microfluidic Sorting in an Optical Lattice, Nature (London) 426, 421 (2003).

[15] T. Laurell, F. Petersson, and A. Nilsson, Chip Integrated Strategies for Acoustic Separation and Manipulation of Cells and Particles, Chem. Soc. Rev. 36, 492 (2007).

[16] H. M. Ji, V. Samper, Y. Chen, C. K. Heng, T. M. Lim, and L. Yobas, Silicon-Based Microfilters for Whole Blood Cell Separation, Biomed. Microdevices 10, 251 (2007).

[17] J. Takagi, M. Yamada, M. Yasuda, and M. Seki, Continuous Particle Separation in a Microchannel Having Asymmetrically Arranged Multiple Branches, Lab Chip 5, 778 (2005).

[18] M. Yamada and M. Seki, Hydrodynamic Filtration for OnChip Particle Concentration and Classification Utilizing Microfluidics, Lab Chip 5, 1233 (2005).

[19] S. Choi and J.K. Park, Continuous Hydrophoretic Separation and Sizing of Microparticles Using Slanted Obstacles in a Microchannel, Lab Chip 7, 890 (2007).

[20] A. Alexeev, R. Verberg, and A.C. Balazs, Patterned Surfaces Segregate Compliant Microcapsules, Langmuir 23, 983 (2007).

[21] K. Smith, A. Alexeev, R. Verberg, and A.C. Balazs, Designing a Simple Ratcheting System to Sort
Microcapsules by Mechanical Properties, Langmuir 22, 6739 (2006).

[22] A. Alexeev, R. Verberg, and A.C. Balazs, Motion of Compliant Capsules on Corrugated Surfaces: A Means of Sorting by Mechanical Properties, J. Polym. Sci. B 44, 2667 (2006).

[23] J.P. Arata and A. Alexeev, Designing Microfluidic Channel That Separates Elastic Particles upon Stiffness, Soft Matter 5, 2721 (2009).

[24] W. Mao and A. Alexeev, Hydrodynamic Sorting of Microparticles by Size in Ridged Microchannels, Phys. Fluids 23, 051704 (2011).

[25] L. R. Huang, E.C. Cox, R.H. Austin, and J.C. Sturm, Continuous Particle Separation through Deterministic Lateral Displacement, Science 304, 987 (2004).

[26] S. Ookawara, R. Higashi, D. Street, and K. Ogawa, Feasibility Study on Concentration of Slurry and Classification of Contained Particles by Microchannel, Chem. Eng. J. (Lausanne) 101, 171 (2004).

[27] D. R. Gossett and D. Di Carlo, Particle Focusing Mechanisms in Curving Confined Flows, Anal. Chem. 81, 8459 (2009).

[28] D. Di Carlo, D. Irimia, R. G. Tompkins, and M. Toner, Continuous Inertial Focusing, Ordering, and Separation of Particles in Microchannels, Proc. Natl. Acad. Sci. U.S.A. 104, 18892 (2007).

[29] J.P. Beech, S. H. Holm, K. Adolfsson, and J. O. Tegenfeldt, Sorting Cells by Size, Shape and Deformability, Lab Chip 12, 1048 (2012).

[30] S. H. Holm, J. P. Beech, M. P. Barrett, and J. O. Tegenfeldt, Separation of Parasites from Human Blood Using Deterministic Lateral Displacement, Lab Chip 11, 1326 (2011).

[31] S. Sugaya, M. Yamada, and M. Seki, Observation of Nonspherical Particle Behaviors for Continuous ShapeBased Separation Using Hydrodynamic Filtration, Biomicrofluidics 5, 024103 (2011).

[32] A. Valero, T. Braschler, A. Rauch, N. Demierre, Y. Barral, and P. Renaud, Tracking and Synchronization of the Yeast Cell Cycle Using Dielectrophoretic Opacity, Lab Chip 11, 1754 (2011).

[33] G. Segré and A. Silberberg, Radial Particle Displacements in Poiseuille Flow of Suspensions, Nature (London) 189, 209 (1961).

[34] D. Di Carlo, J. F. Edd, D. Irimia, R. G. Tompkins, and M. Toner, Equilibrium Separation and Filtration of Particles Using Differential Inertial Focusing, Anal. Chem. 80, 2204 (2008).

[35] D. Di Carlo, Inertial Microfluidics, Lab Chip 9, 3038 (2009).

[36] A.A.S. Bhagat, S.S. Kuntaegowdanahalli, and I. Papautsky, Inertial Microfluidics for Continuous Particle Filtration and Extraction, Microfluid. Nanofluid. 7, 217 (2008).

[37] S.C. Hur, H.T.K. Tse, and D. Di Carlo, Sheathless Inertial Cell Ordering for Extreme Throughput Flow Cytometry, Lab Chip 10, 274 (2010).

[38] A.J. Mach and D. Di Carlo, Continuous Scalable Blood Filtration Device Using Inertial Microfluidics, Biotechnol. Bioeng. 107, 302 (2010). 
[39] A. J. Mach, J.H. Kim, A. Arshi, S.C. Hur, and D. Di Carlo, Automated Cellular Sample Preparation Using a Centrifuge-on-a-Chip, Lab Chip 11, 2827 (2011).

[40] S. C. Hur, N. K. Henderson-MacLennan, E. R. B. McCabe, and D. Di Carlo, Deformability-Based Cell Classification and Enrichment Using Inertial Microfluidics, Lab Chip 11, 912 (2011).

[41] S. C. Hur, S. E. Choi, S. Kwon, and D. Di Carlo, Inertial Focusing of Non-Spherical Microparticles, Appl. Phys. Lett. 99, 044101 (2011).

[42] F. P. Bretherton, G. F. Carrier, and M S. Longuet-Higgins, Report on the I.U.T.A.M. Symposium on Rotating Fluid Systems, J. Fluid Mech. 26, 393 (1996) [published online 2006].

[43] S. Yang, J.-Y. Kim, S.-J. Lee, S. S. Lee, and J.M Kim, Sheathless Elasto-Inertial Particle Focusing and Continuous Separation in a Straight Rectangular Microchannel, Lab Chip 11, 266 (2011).

[44] G. B. Jeffery, The Motion of Ellipsoidal Particles Immersed in a Viscous Fluid, Proc. R. Soc. A 102, 161 (1922).

[45] C. Pozrikidis, Orbiting Motion of a Freely Suspended Spheroid near a Plane Wall, J. Fluid Mech. 541, 105 (2005).

[46] S. R. Keller and R. Skalak, Motion of a Tank-Treading Ellipsoidal Particle in a Shear Flow, J. Fluid Mech. 120, 27 (1982) [published online 2006].

[47] D. L. Koch and R. J. Hill, Inertial Effects in Suspension and Porous-Media Flows, Annu. Rev. Fluid Mech. 33, 619 (2001).

[48] G. Subramanian and D. L. Koch, Centrifugal Forces Alter Streamline Topology and Greatly Enhance the Rate of Heat and Mass Transfer from Neutrally Buoyant Particles to a Shear Flow, Phys. Rev. Lett. 96, 134503 (2006).

[49] See Supplemental Material at http://link.aps.org/ supplemental/10.1103/PhysRevX.2.031017 for simulations of equilibrium orientations, movies, and supplemental figures.

[50] D. Di Carlo, J. F. Edd, K. J. Humphry, H. A. Stone, and M. Toner, Particle Segregation and Dynamics in Confined Flows, Phys. Rev. Lett. 102, 094503 (2009).

[51] D. Petranovic and G. N. Vemuri, Impact of Yeast Systems Biology on Industrial Biotechnology, J. Biotechnol. 144, 204 (2009).

[52] G. M. Walker, Synchronization of Yeast Cell Populations, Methods in Cell Science 21, 87 (1999).

[53] K. Shedden and S. Cooper, Analysis of Cell-Cycle Gene Expression in Saccharomyces cerevisiae Using
Microarrays and Multiple Synchronization Methods, Nucleic Acids Res. 30, 2920 (2002).

[54] M. Nikolic-Jaric, G. A. Ferrier, S. Rzeszowski, T. Cabel, S. Nandagopal, F. Lin, G. E. Bridges, D. J. Thomson, and M. R. Freeman, in Proceedings of Micro Total Analysis Systems (MicroTAS) 2010, Groningen, The Netherlands, http://www.rsc.org/binaries/LOC/2010/PDFs/Papers/457_ 0955.pdf, pp. 1340-1342.

[55] D. Spencer and H. Morgan, Positional Dependence of Particles in Microfluidic Impedance Cytometry, Lab Chip 11, 1234 (2011).

[56] S.C. Chapin, D.C. Pregibon, and P.S. Doyle, HighThroughput Flow Alignment of Barcoded Hydrogel Microparticles, Lab Chip 9, 3100 (2009).

[57] S. H. Lee, S.E. Choi, A. J. Heinz, W. Park, S. Han, Y. Jung, and S. Kwon, Active Guidance of $3 D$ Microstructures, Small 6, 2668 (2010).

[58] E. Sollier, C. Murray, P. Maoddi, and D. Di Carlo, Rapid Prototyping Polymers for Microfluidic Devices and High Pressure Injections, Lab Chip 11, 3752 (2011).

[59] M. B. Elowitz, A. J. Levine, E. D. Siggia, and P. S. Swain, Stochastic Gene Expression in a Single Cell, Science 297, 1183 (2002).

[60] J.A. Champion and S. Mitragotri, Role of Target Geometry in Phagocytosis, Proc. Natl. Acad. Sci. U.S.A. 103, 4930 (2006).

[61] W. Lee, H. Amini, H. A. Stone, and D. Di Carlo, Dynamic Self Assembly and Control of Microfluidic Particle Crystals, Proc. Natl. Acad. Sci. U.S.A. 107, 22413 (2010).

[62] A. Alexeev, R. Verberg, and A.C. Balazs, Modeling the Motion of Microcapsules on Compliant Polymeric Surfaces, Macromolecules 38, 10244 (2005).

[63] S. Succi, The Lattice Boltzmann Equation for Fluid Dynamics and Beyond (Oxford University Press, Oxford, 2001).

[64] A. J.C. Ladd and R. Verberg, Lattice-Boltzmann Simulations of Particle-Fluid Suspensions, J. Stat. Phys. 104, 1191 (2001).

[65] M. Bouzidi, M. Firdaouss, and P. Lallemand, Momentum Transfer of a Boltzmann-Lattice Fluid with Boundaries, Phys. Fluids 13, 3452 (2001).

[66] H. Masoud and A. Alexeev, Modeling Magnetic Microcapsules That Crawl in Microchannels, Soft Matter 6, 794 (2010).

[67] A. Kilimnik, W. Mao, and A. Alexeev, Inertial Migration of Deformable Capsules in Channel Flow, Phys. Fluids 23, 123302 (2011). 